\title{
Study of the Hadronization Dynamics with Nuclei
}

\section{Raphaël Dupré* on behalf of the CLAS collaboration}

CEA, Centre de Saclay, Irfu/Service de Physique Nucléaire, 91191 Gif-sur-Yvette, France

E-mail: raphael.dupre@cea.fr

\begin{abstract}
Hadronization happens at the femtometer scale. Therefore, comparing nuclei of various sizes is the best way to study this process. Experiments based on a broad range of reactions, like semi-inclusive deep inelastic scattering (SIDIS), Drell-Yan and heavy ion collisions, have already permitted to understand a lot about in-medium hadronization. In this presentation, we will review the models describing nuclear SIDIS and put them into perspective with our analysis of the recent data from Hall B at Jefferson Laboratory (CLAS Collaboration). The impact of these high precision data, and their ability to discriminate between existing models, will be discussed. Finally, future prospects and challenges will be addressed.
\end{abstract}

Sixth International Conference on Quarks and Nuclear Physics,

April 16-20, 2012

Ecole Polytechnique, Palaiseau, Paris

\footnotetext{
${ }^{*}$ Speaker.
} 


\section{Introduction}

The hadronization of quarks, which is the focus of this presentation, has a very limited theoretical understanding (see [1] for a review). In order to be modeled, the process can be separated in two stages, as sketched in Fig. 1. The production time corresponds to the period after the interaction, when the colored quark propagates and emits gluons. The formation time corresponds to the time needed for a pre-hadron to become a fully formed hadron.

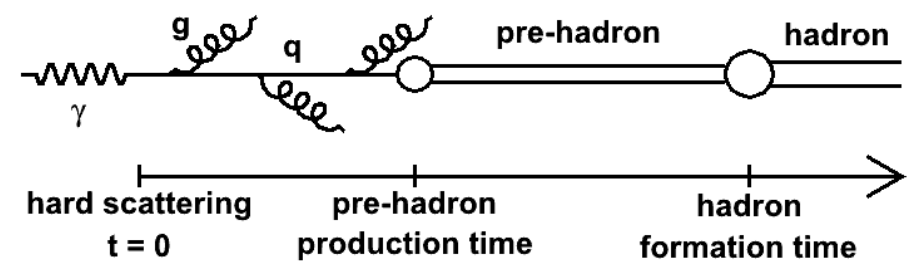

Figure 1: The hadronization of a quark from DIS illustrating the production and formation time.

In order to understand the dynamics of hadronization, we need to be able to quantify these characteristic times and understand the behavior and evolution of the quarks and pre-hadrons in nuclear matter. This leads to the study of important processes, such as color dipole propagation in nuclear matter. Quark propagation is also an interesting means to characterize a QCD medium and has been used extensively for both cold and hot nuclear matter [2,3]. Finally, understanding hadronization in the nuclear medium is of importance in order to reduce systematics when correcting for it. Since many experiments (neutrino-nuclei typically) measure hadrons produced on nuclear targets, in order to increase their cross section, they need to correct for hadronization effects.

To characterize the hadronization in nuclei, we focus on the multiplicity ratio observable. It is directly linked to the attenuation of the hadrons in the medium (which is $1-R_{A}^{h}$ ) and is defined as

$$
R_{A}^{h}\left(Q^{2}, v, z, P_{T}^{2}\right)=\frac{N_{A}^{h}\left(Q^{2}, v, z, P_{T}^{2}\right) / N_{A}^{e}\left(Q^{2}, v\right)}{N_{D}^{h}\left(Q^{2}, v, z, P_{T}^{2}\right) / N_{D}^{e}\left(Q^{2}, v\right)}
$$

with $N_{A}^{h}$ the number of hadrons $h$ produced on a target $A$ and $N_{A}^{e}$ the number of electrons scattered from the target $A$. Based on Fig. 2, the usual kinematics factors are defined as follow: $Q^{2}=-q^{2}$, the 4-momentum squared of the virtual photon; $v=p \cdot q / M_{n}$, the energy of the virtual photon in the target rest frame (with $M_{n}$ the mass of the nucleon); $z=p_{h} \cdot p / q \cdot p$, the fractional energy carried by the hadron and $P_{T}^{2}$, the transverse momentum squared of the hadron, with respect to the virtual photon.

\section{Previous Measurements}

Numerous measurements were done in the past for a wide range of nuclei and kinematics. We highlight here the recent results from HERMES, which showed that all three pions behave similarly. Fig 3 illustrates the general picture observed for the multiplicity ratio of pions as a function of various kinematical variables. As a function of $v$, the Lorentz boost experienced by the system leads to a reduced absorption at higher energy. Indeed, a faster quark will have less time to 


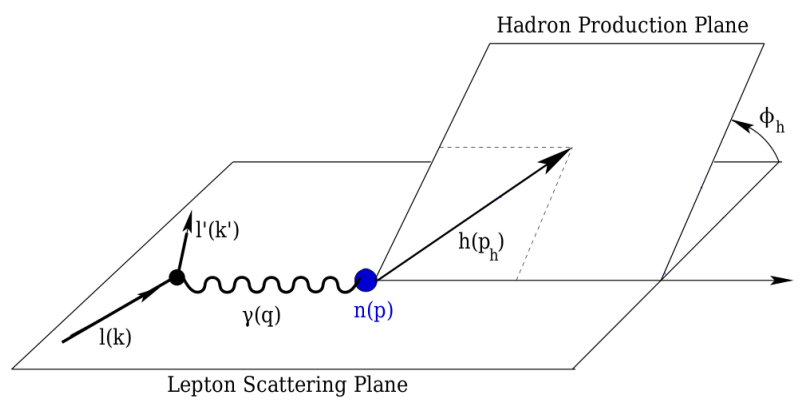

Figure 2: Schematic diagram of a SIDIS event.

emit gluons in the medium [4] and will hadronize farther out, reducing the hadronic interaction in the medium [5]. As a function of $z$, the ratio strongly decreases, this phenomena has two competing explanations, either the production time is shorter at high $z$ [5], leading to an increased path in the nucleus for the (pre-)hadron, or the behavior of the fragmentation functions, which drop faster at high $z$, leads to a larger relative effect [4] from quark energy loss in the medium. As a function of $Q^{2}$, a small effect is observed, however it may be due to the correlation of this variable with $v$. Yet, this effect could be linked to the parton energy loss, for which some models predict a small $Q^{2}$ dependence [6]. Finally, as a function of $P_{T}^{2}$, we observe a rise, stronger for the heavier nuclei, which is due to multiple soft scattering populating the high $P_{T}^{2}$ region.

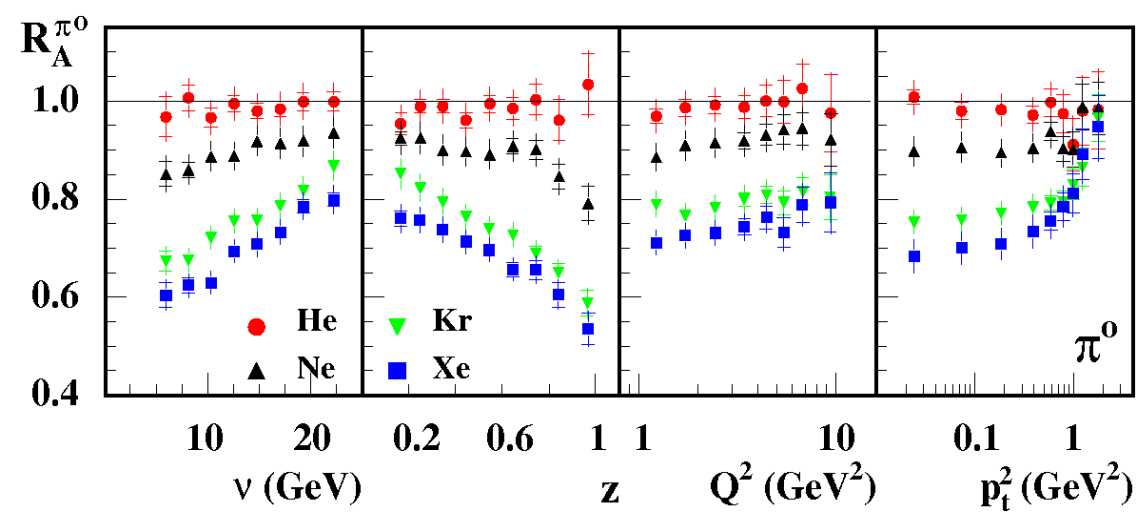

Figure 3: HERMES [7] results for the $\pi^{0}$ multiplicity ratios. The colors are for the different nuclei He (red), $\mathrm{Ne}$ (black), Kr (green) and Xe (blue).

Several other results from HERMES are noteworthy, but have poor statistics or contamination from target fragmentation. Yet HERMES observed interesting hints for unexpected $Q^{2}$ and $A$ dependence, as well as a different behavior for the two charged kaons. Moreover, models built exclusively on parton energy loss or pre-hadron absorption are still compatible with the data. The deduced physical properties of nuclei and pre-hadrons remain model dependent. This motivates a more systematic confrontation of models with the large data set provided by the HERMES collaboration. 


\section{CLAS Results}

The CLAS collaboration at Jefferson Laboratory (JLab) ran an experiment [8] for 50 days with a high luminosity $5 \mathrm{GeV}$ electron beam hitting nuclear targets (D, C, Al, Fe, Sn and $\mathrm{Pb}$ ). The main goal was to collect high statistics for a precise multi-dimensional study of hadronization. Some results of this experiment, obtained in the analysis of charged pions, are presented in this section.

In Fig. 4, the multiplicity ratio is presented as a function of $z$ and $p_{T}^{2}$, which shows the expected behavior with a very strong effect for $p_{T}^{2}$. However, this result is moderated by Monte-Carlo simulation [9], which shows that a significant effect is generated by the Fermi motion of the nucleons. In order to isolate the effect of hadronization, we use carbon instead of deuterium as the reference nuclei. In this case, Fermi motion differences between the nuclei is reduced and Fig 5 (left) shows, as expected, a smaller effect. This method provides, at CLAS energies, results that are easier to compare directly to theory and it should be used whenever possible.

The CLAS experiment also explored a very large range in $A$, from $A=2$ to $A=208$. This large coverage helps to reveal the $A$ dependence of the multiplicity ratio. Fig. 5 (right) shows that it is not linear with $A^{1 / 3}$, i.e. it is not proportional to the path length. The observed result, an attenuation of the nuclear effect with larger nuclei size, is unexpected for most parton energy loss or (pre-)hadron
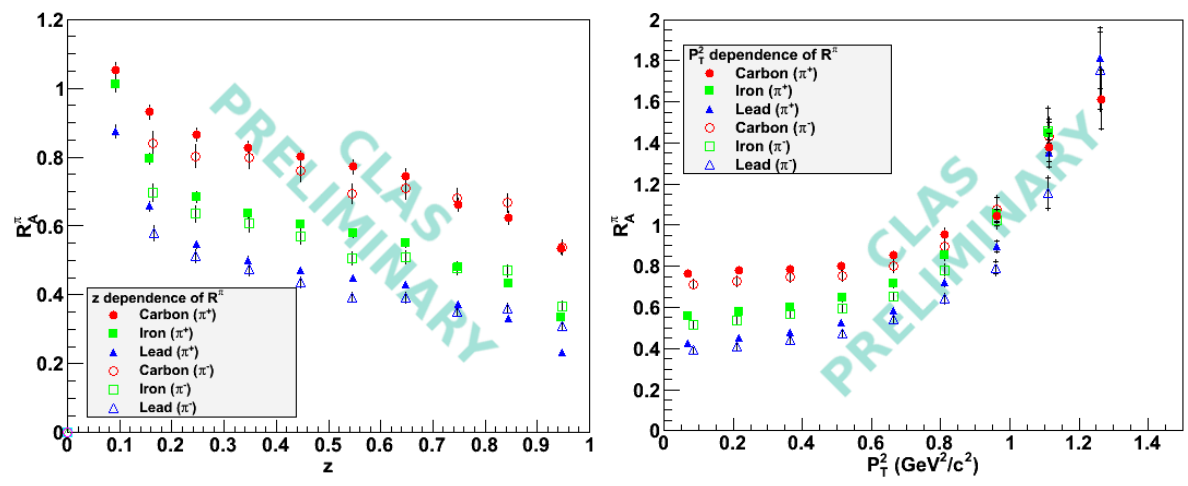

Figure 4: Preliminary results for the multiplicity ratio of charged pions as a function of $z$ (left) and $P_{T}^{2}$ (right). Carbon (red), iron (green) and lead (blue) are presented for $\pi^{+}$(full) and $\pi^{-}$(empty).
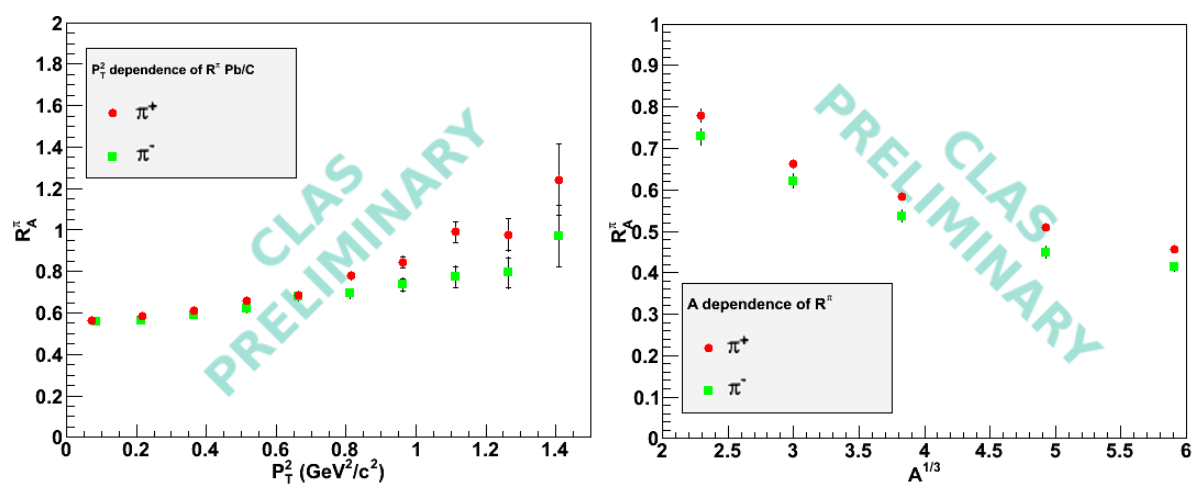

Figure 5: (Left) Preliminary results for the multiplicity ratio of lead normalized to carbon as a function of $P_{T}^{2}$ for $\pi^{+}$(red) and $\pi^{-}$(green). (Right) Preliminary results for the multiplicity ratio as a function of $A^{1 / 3}$ (right) for $\pi^{+}$(red) and $\pi^{-}$(green). 
absorption models, which both predict a dependence on $A^{\alpha}$ with $\alpha \geq 1 / 3$, the opposite of our measurement. One way to solve this problem is to assume that the production time occurs when the quark is still inside the nuclei and that the attenuation observed is due to parton energy loss in the nucleus. In this case, increasing the size has only a small effect when the production length is already smaller than the nuclear size. This result is important to sort out models of hadronization.

\section{Hadronization in the Future}

Several projects exist to study hadronization in future facilities. An experiment [10] is already approved to run in Hall B at JLab after the beam upgrade to $12 \mathrm{GeV}$. This measurement will be especially suited to measure the production and formation times for kinematics between CLAS and HERMES. Fig. 6 illustrates the coverage in $v$ and $Q^{2}$ of this proposal.

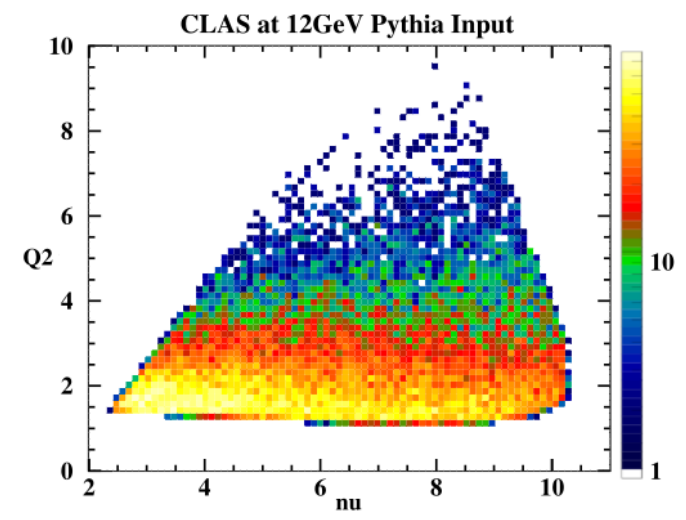

Figure 6: Kinematic coverage in $Q^{2}\left(\mathrm{GeV}^{2}\right)$ and $v(\mathrm{GeV})$ achievable by a hadronization measurement with CLAS12 [10].

Further in the future, the Electron-Ion Collider (EIC) project gives important opportunities to explore parton energy loss in the purely perturbative QCD regime. The machines foreseen by JLab and RHIC provide a maximum center of mass energy $s \sim 1000 \mathrm{GeV}^{2}$, with the possibility to run at lower energy, and high luminosity $\left(\sim 10^{34} \mathrm{~cm}^{2} \mathrm{~s}^{-1}\right)$. The high energy will enable one to reach a production time far outside the nuclei and to go at high $Q^{2}$, where perturbative QCD applies. This opens many new opportunities. First, there is the possibility to cover $Q^{2}$ on two orders of magnitude with good accuracy (see projections in Fig. 7 (left)). This large coverage in $Q^{2}$ seems to be the best way to accurately measure the small nuclear effect expected. Second, detecting heavy mesons, in particular charmed ones, is possible with the EIC (see projections in Fig. 7 (right)), which will allow us to compare cold nuclear results with the ones already obtained for hot nuclear matter $[11,12]$. This measurement is especially interesting since both RHIC and LHC results have revealed unexpected behaviors. Many other measurements are possible to study hadronization and parton energy loss at an EIC, which are described in [13].

\section{Conclusion}

Hadronization is an important topic for which the theoretical description is still a turmoil. Therefore, clean experimental measurements are needed in order to understand its space-time evo- 

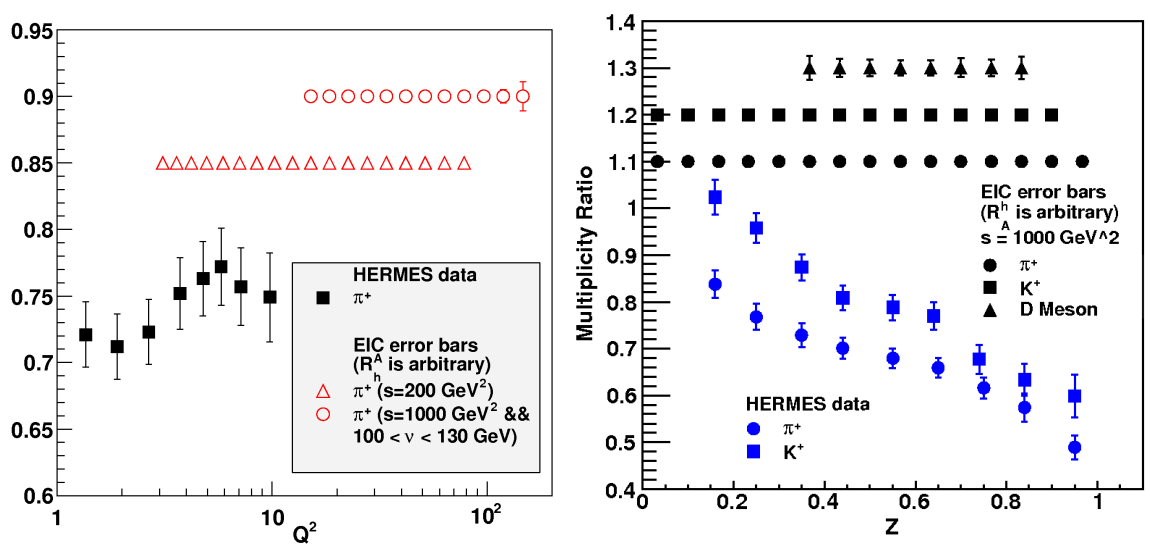

Figure 7: Error estimation for a multiplicity ratio measurement at an EIC. (Left) Coverage as a function of $Q^{2}\left(\mathrm{GeV}^{2}\right)$ for $s=200 \mathrm{GeV}^{2}$ and $s=1000 \mathrm{GeV}^{2}$. (Right) Different hadrons, characterizing different quarks, as a function of $z$. These projections are compared with results from [7].

lution and its properties in nuclear matter. The CLAS data, and in particular the results presented here, show the important effect due to Fermi motion of nucleons. They also provide stringent tests to discriminate between the different possible mechanisms, giving a preference to parton energy loss over pre-hadron absorption. We finally presented some future plans to study hadronization with the medium energy JLab $12 \mathrm{GeV}$ beam and a high energy EIC.

\section{References}

[1] A. Accardi, F. Arleo, W. K. Brooks, D. D’Enterria and V. Muccifora, Riv. Nuovo Cim. 32 (2010) 439 [arXiv:0907.3534 [nucl-th]].

[2] E. Wang and X.-N. Wang, Phys. Rev. Lett. 89 (2002) 162301 [hep-ph/0202105].

[3] D. d'Enterria, arXiv:0902.2011 [nucl-ex].

[4] F. Arleo, Eur. Phys. J. C 30 (2003) 213 [hep-ph/0306235].

[5] K. Gallmeister and U. Mosel, Nucl. Phys. A 801 (2008) 68 [nucl-th/0701064].

[6] B. Z. Kopeliovich, J. Nemchik, E. Predazzi and A. Hayashigaki, Eur. Phys. J. A 19S1 (2004) 111.

[7] A. Airapetian et al. [HERMES Collaboration], Nucl. Phys. B 780 (2007) 1 [arXiv:0704.3270 [hep-ex]].

[8] W. Brooks et al., Proposal to Jefferson Lab PAC 22 (2002).

[9] R. Dupré and A. Accardi, To be published.

[10] K. Hafidi et al., Proposal to Jefferson Lab PAC 30 (2006).

[11] S. S. Adler et al. [PHENIX Collaboration], Phys. Rev. Lett. 96 (2006) 032301 [nucl-ex/0510047].

[12] B. Abelev et al. [ALICE Collaboration], arXiv:1203.2160 [nucl-ex].

[13] D. Boer, M. Diehl, R. Milner, R. Venugopalan, W. Vogelsang, D. Kaplan, H. Montgomery and S. Vigdor et al., arXiv:1108.1713 [nucl-th]. 